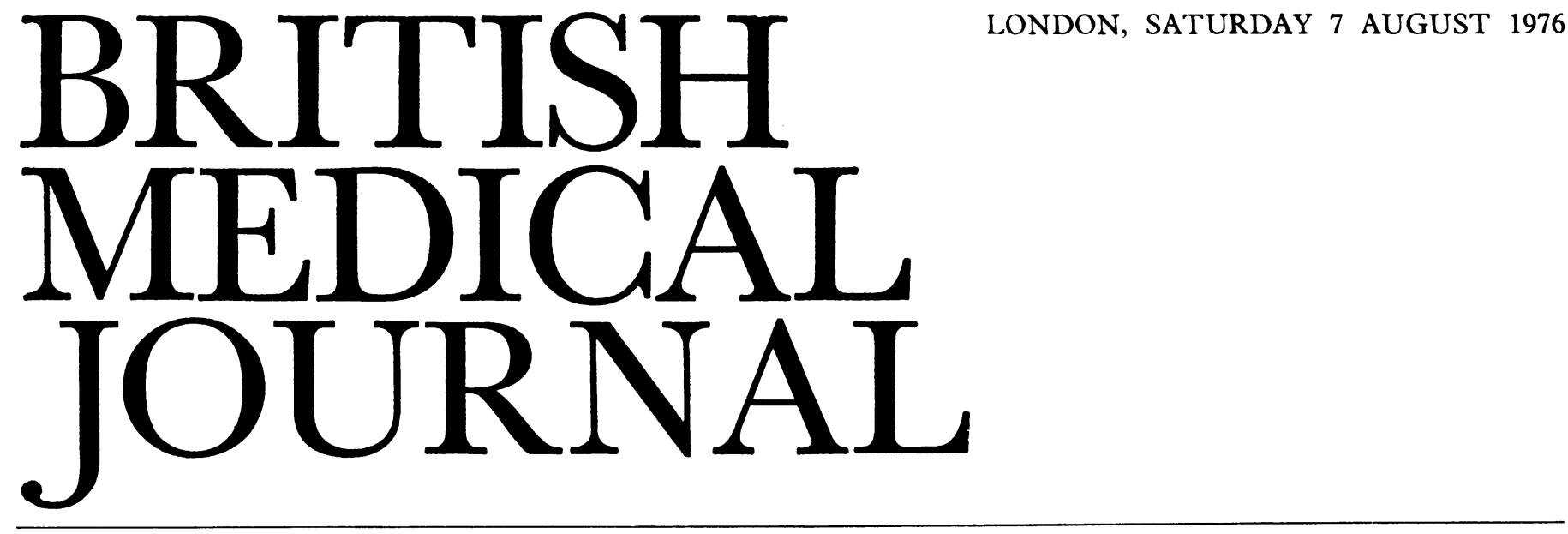

Most episodes of food poisoning are traceable to contaminated meat and poultry, but the variety of other foodstuffs implicated includes such unusual and seemingly innocuous items as French cheese, ${ }^{1}$ chocolate, ${ }^{2}$ black pepper, ${ }^{3}$ cider,${ }^{4}$ and salami. ${ }^{5}$ Salmonellas and Clostridium welchi are common contaminants of poultry and meat, ${ }^{6}$ and Vibrio parahaemolyticus of seafood. Bacillus cereus, commonly found in soil and vegetation, a more recently described cause of food poisoning, has been detected in $88 \%$ of samples of uncooked rice ${ }^{7}$ and, variously, in $40 \%$ and $72 \%$ of samples of spices. ${ }^{8}{ }^{9}$

Summer brings to many of us a general feeling of wellbeing, and micro-organisms, too, relish the warmth; but whereas humans have the choice of several other pursuits the mcre lowly bacterium has the resources only to reproduce. This it can do very well given the right conditions, and organisms can reach levels high enough to cause food poisoning rapidly, especially when foods such as cooked meat and poultry are stored unrefrigerated in warm weather. The number of foodpoisoning incidents tends to rise at such times, and over half the 10655 cases notified to the Registrar General in 1975 were in the four months between June and September. This somewhat high total for the year represents an increase of $39 \%$ on that ${ }^{10}$ for 1974 and could well have been related to the unusually warm summer of 1975 . The organisms primarily responsible for this seasonal rise are the salmonellas-which account for about $80 \%$ of cases in which a micro-organism is identifiedstaphylococci, and $B$ cereus. ${ }^{11-13} C$ welchi outbreaks do not appear to have a consistent seasonal variation. ${ }^{11}$

The essential rule is to avoid storing foods such as cooked meat, poultry, seafoods, and rice at temperatures at which bacteria proliferate. Most food poisoning bacteria grow in the range $8-50^{\circ} \mathrm{C}$. The range of optimum growth, $30-47^{\circ} \mathrm{C}$, is quite wide, but it varies with different organisms, ${ }^{1415} C$ welchi apparently multiplying best at $43-47^{\circ} \mathrm{C}$ and $B$ cereus at $30-37^{\circ} \mathrm{C}$, while staphylococci and salmonellas have a more restricted range around human body temperature, as does $V$ parahaemolyticus. ${ }^{16}$ Temperatures above $60^{\circ} \mathrm{C}$ will destroy most vegetative cells, but even boiling may not destroy the toxins of Staphylococcus aureus or the spores of $B$ cereus or $C$ welchi. Food must therefore be served shortly after cooking or else cooled rapidly and stored in an ordinary domestic refrigerator regulated to $4{ }^{\circ} \mathrm{C}$ within about an hour. ${ }^{17}$ This time period is important because the vegetative cells of $C$ welchi, for example, can divide as frequently as every 10 to 12 minutes in optimum conditions during the logarithmic phase. ${ }^{14}$

Other practices may encourage the spread of bacteria. One is slicing or mincing meat or poultry while still warm-every cut in meat spreads contamination. Indeed, minced meat seems to encourage bacterial growth, and normal bacterial flora multiply almost a hundredfold in fresh minced meat even when stored at about $1^{\circ} \mathrm{C}$ for 48 hours, compared with about threefold in $100 \mathrm{~g}$ chunks of meat. ${ }^{18}$ Nevertheless cooking too large a bulk of food should also be discouraged: meat and poultry should be cooked in pieces no larger than $3 \mathrm{~kg}(6.6 \mathrm{lb}){ }^{17}$ If cooking and storage of a large volume of food is unavoidable it should be consumed soon after cooking or divided into smaller portions for cooling and storage. If left to cool in bulk the inside of the mass of food will remain warm long enough for bacteria to multiply to dangerous levels. In particular, the spores of $C$ welchi and $B$ cereus will germinate and grow. This appears to be a common error in cooking rice in some Chinese restaurants, where fried rice is made from boiled rice cooked in bulk and stored unrefrigerated for lengthy periods. ${ }^{13}$ Outbreaks of $B$ cereus food poisoning, almost always from Chinese fried rice, are now commonly reported. ${ }^{13}$

Another hazard to avoid in the treatment of food after cooking is contamination or recontamination from organisms that may be present in raw foods or on surfaces or utensils. Raw foods, especially meat, must be kept quite separate from cooked, and different surfaces should be set aside for each. If this is not possible, surfaces and utensils used for raw meat must be washed down thoroughly with hot water and detergent immediately after use. This is of particular importance when eviscerating poultry. The combination of contamination and inadequate storage after cooking is one of the most common errors leading to outbreaks of food poisoning in Britain today. It is important to remember that contamination of cooked foods may also arise from man. Both salmonellas and staphylococci in skin lesions or in the nose may be transmitted in this way.

Food may be seriously contaminated but yet remain wholesome to our human senses of smell, taste, and vision. "There is no art to find the mind's construction in the face," and the outward show of a restaurant is not a reflection of its kitchen. Buying cooked meat from shops that do not keep utensils, counters, personnel, and trays for raw and cooked meat, fish, 
or poultry strictly separate is hazardous and it is advisable to take one's custom elsewhere.

In summer most of us want to spend as little time as possible over a hot stove, and there is a temptation to store food for lengthy periods after it has been cooked. Cold meat and poultry, sandwiches, and buffet meals are popular at this time of year, and summer is also the time for picnics. A few warm hours in the boot of a car may be sufficient to allow foodpoisoning bacteria to multiply to levels high enough to cause food poisoning. But if food is properly refrigerated as described -and, for picnics, insulated cooler bags and ice boxes are available-the risk of food poisoning should decrease.

${ }^{1}$ Marier, R, et al, Lancet, 1973, 2, 1376.

2 Craven, $\mathrm{P}$ C, et al, Lancet, 1975, 1, 788

${ }^{3}$ Epidemiological Bulletin, Health and Welfare Canada, 1974, 18(4), 62.

4 US Center for Disease Control, Morbidity and Mortality, 1975, 24, 87.

5 US Center for Disease Control, Morbidity and Mortality, 1975, 24, 374

${ }^{6}$ Roberts, D, fournal of Hygiene, 1972, 70, 565.

${ }^{7}$ Gilbert, R J, and Taylor, A J, Archiv für Lebensmittelhygiene, 1975, 26, 38

${ }^{8} \mathrm{Kim}, \mathrm{H}$ U, and Goepfert, J M, fournal of Milk and Food Technology, 1971, 34, 12.

9 Nygren, B, Acta Pathologica et Microbiologica Scandinavica, Supplementum, 1962, No 160 .

10 OPCS Monitor, Registrar General's Weekly Return for England and Wales, No 52. London, HMSO, 1976

11 Vernon, E, and Tillett, H E, Public Health, London, 1973/1974, 88, 225.

12 Thomas, M E M, and Tillett, H E, fournal of Hygiene, 1975, 74, 183.

13 Public Health Laboratory Service, British Medical fournal, 1973, 3, 647.

${ }^{14}$ Hobbs, B C, Food Poisoning and Food Hygiene, 3rd edn. London, Edward Arnold, 1974.

${ }^{15}$ Gilbert, R J, Stringer, M F, and Peace, T C, Fournal of Hygiene, 1974, 73, 433.

${ }^{16}$ Sakazaki, R, in The Microbiological Safety of Food, eds B C Hobbs and J H B Christian. New York and London, Academic Press, 1973.

17 Noah, N D, British Medical fournal, 1975, 4, 714.

18 Hess, E, in The Microbiological Safety of Food, eds B C Hobbs and J H B Christian. New York and London, Academic Press, 1973.

\section{Adjuvant chemotherapy in colorectal cancer}

The overall five-year cure rate of cancer of the large bowel has not changed much in the last ten years. In about a quarter of the patients at diagnosis the tumour will be found to have spread beyond the reach of surgery, though in most instances some form of palliative resection is possible. Where curative resection seems possible the probability of disseminated subclinical micrometastases is correlated with the extent of the local disease.

In the last decade many surgical teams have tried to improve survival after colorectal cancer by using chemotherapy as an adjuvant to surgery. The sheet anchor has been 5-fluorouracil (5FU), but so far the results of trials have been disappointing. Rousselot et $a l^{1}$ reported that the combination of intraluminal instillation of 5FU during surgery and a brief course of the drug intravenously gave an improved survival in patients with positive nodes (Dukes $\mathrm{C}$ lesions): in their series 56\% were alive at eight years as compared with an expected $32 \%$ at five years. Lawrence $e t a^{2}$ in a carefully controlled study supplemented this regimen with five courses of oral $5 \mathrm{FU}$ in the first year after surgery. Their analysis showed no clinically significant benefit for the treated group. This inability to influence the evolution of micrometastases of colorectal cancer has been confirmed by the Veterans Administration Surgical Adjuvant Cancer Chemotherapy Group, whose interim data suggest that extending the intermittent courses of 5FU until the 19th month after surgery still does not help. This disappointment contrasts with their earlier study, which suggested some advantage for patients given $5 \mathrm{FU}$, but at a level not reaching statistical significance.

When given alone for the treatment of colorectal cancer the real value of $5 \mathrm{FU}$ is difficult to assess: somewhere between $15 \%$ and $20 \%$ of patients with advanced disease may expect an objective response from treatment with an adequate dose $\left(500 \mathrm{mg} / \mathrm{m}^{2}\right.$ intravenously daily for five days repeated every six weeks). This is more aggressive treatment and far more demanding in resources than seems to have been used in the past as an adjuvant programme.

We are still waiting for a really effective agent or combination of agents to combat the growth of metastatic colorectal cancer. On balance the experience of the major centres suggests that a combination of methyl CCNU (one of the nitrosourea family not yet available on prescription in Britain) and 5FU is the most reliable mixture, but, even so, benefit may be expected in only $30 \%$ of cases of well-established tumours ${ }^{3}$ and there have been no evaluable data on the use of this combination as an adjuvant. Moertel ${ }^{4}$ reported a $43 \%$ response rate when vincristine was added to this combination.

It is an axiom that the benefit of any adjuvant chemotherapy programme should not be outweighed by the burden of drug toxicity. Cytotoxic drug treatment may result in greater misery than that produced by the cancer and at its worst the premature death of the patient from bone marrow failure at a time when the metastases might have been causing little or no deterioration in wellbeing. The aggressive and more effective treatments always carry the risk of toxicity, and some patients will show unexpected sensitivity to their potentially lethal side effects. Ideally, therefore, the strategy should be to restrict treatment to those patients in whom there is persuasive evidence, either direct or indirect, of early metastatic cancer. This group should include patients in whom the surgeon was aware that there was some tumour left at the excision site, on the peritoneum, or in the liver.

A careful inspection of the abdomen at laparotomy and of the resected specimen is still the best guide to the patient's immediate prognosis. This clinical assessment may be aided by measuring the appropriate markers, such as carcinoembryonic antigen (CEA) and sensitive indicators of liver function. A persistently high and rising blood concentration of CEA after resection virtually always means disseminated disease; while serial measurement of CEA every three months in high-risk patients may provide good evidence of recurrent disease before clinical surveillance does. This information might reduce the unfavourable effects of beginning chemotherapy too late in those with micrometastases while helping to protect those patients with Dukes C or B lesions who had been cured by surgery from the hazards of unnecessary aggressive chemotherapy. A trial of this type could be mounted in England and Wales using the resources of the supraregional assay service to monitor CEA values and of the growing numbers of medical and surgical oncologists willing and able to work within the constraints of a strict protocol.

Alternatively, an attempt might be made to find out whether some form of immunotherapy might be of benefit when given to patients with minimal tumour and those with an apparent curative resection of a Dukes B or C lesion with a postoperative blood CEA concentration in the normal range. Levamasole treatment seems possibly to delay the appearance of metastases in breast cancer, and its place needs further study, while the methanol-extracted residue of BCG (MER) is being tested extensively by the National Cancer Institute. MER may have sufficient potency, without risk, to warrant a large scale trial. 